\title{
THE EFFECT OF SENSOR FAILURE ON THE ATTITUDE AND RATE ESTIMATION OF THE MAP SPACECRAFT
}

\author{
Itzhack Y. Bar-Itzhack \\ Faculty of Aerospace Engineering \\ Technion-Israel Institute of Technology \\ Haifa 32000 , Israel \\ Richard R. Harman" \\ Flight Dynamics Analysis Branch, Code 595 \\ Mission Engineering and Systems Analysis Division \\ NASA-Goddard Space Flight Center \\ Greenbelt, MD 20771, USA
}

\begin{abstract}
This work describes two algorithms for computing the angular rate and attitude in case of a gyro and a Star Tracker failure in the Microwave Anisotropy Probe (MAP) satellite, which was placed in the L2 parking point from where it collects data to determine the origin of the universe. The nature of the problem is described, two algorithms are suggested, an observability study is carried out and real MAP data are used to determine the merit of the algorithms. It is shown that one of the algorithms yields a good estimate of the rates but not of the attitude whereas the other algorithm yields a good estimate of the rate as well as two of the three attitude angles. The estimation of the third angle depends on the initial state estimate. There is a contradiction between this result and the outcome of the observability analysis. An explanation of this contradiction is given in the paper. Although this work treats a particular spacecraft, its conclusions are more general.
\end{abstract}

\section{INTRODUCTION}

The Microwave Anisotropy Probe (MAP) Satellite was launched at 15:46:46 EDT on June 30,2001 aboard a Delta II-7425-10 launch vehicle. After three phasing loops MAP passed by a swingby point where it was injected by the moon's gravity towards the L2 point. On October 1, 2001, following a three month journey, MAP arrived safely at its permanent observing station near the L2 Lagrange Point, 1.5 million $\mathrm{km}$ from Earth, a quasi-stable position a million miles from Earth in the direction opposite the Sun.

MAP used two two-axis gyroscopes to measure its angular rate vector. One input axis of the first gyroscope was aligned along the body $x$-axis and the other input axis was

- Sophie and William Shamban Professor of Aerospace Engineering. Member Technion Asher Space Research Institute. This work was done as an NRC NASA Resident Research Associate. Tel: +972 4 829-3196, Fax: +9724 829-2030, Email: ibaritz@technion.ac.il

'Aerospace Engineer. Tel: (301) 286-5125, Fax: (301) 286-0369, Email: richard.r.harman@nasa.gov aligned along the body $z$-axis. The input axes of the second gyro were aligned along the body $y$ and $z$-axes. Therefore, if one gyro failed then the rate about two body axes is still measured; namely, about the $z$-axis and one of the other two. MAP also had two Digital Sun Sensors that measured one vector; namely, the direction to the Sun. In addition, the spacecraft also carried two Star Trackers. However, when MAP performed the phasing loops it passed through the Van-Allen radiation belts, where the star trackers were inoperative.

The problem handled in this paper is as follows. Suppose that indeed one gyro failed and the star trackers were inactive when MAP passed through the Van-Allen belts. Was it still possible to reconstruct the full angular rate and the orientation of MAP with the one operating gyro and a measurement of the Sun direction? It should be noted that although this question concerns a particular mission, its implication is more general.

We addressed this problem by defining two possible filtering models and performing a nonlinear observability analysis. Then we performed simulations of the spacecraft motion and ran the suitable filters to verify the results of the analysis. In the next section we describe the angular rate at which the problem is examined. This section is followed by a description of the examined filters. In the section that follows we present the observability analysis that was carried out. In the next section we present simulation test results and then, in the last section, we present our conclusions.

\section{Body Angular Kinematics}

During the critical part of the trajectory; that is, when MAP passed through the radiation belts the spacecraft was kept at an inertial hold where the angular rate was nominally zero. In this mode the spacecraft attitude control system acted as a regulator where the gyros nominally read no input. In order to examine our suggested filters under more stringent conditions we chose to examine the filters under non-zero nominal 


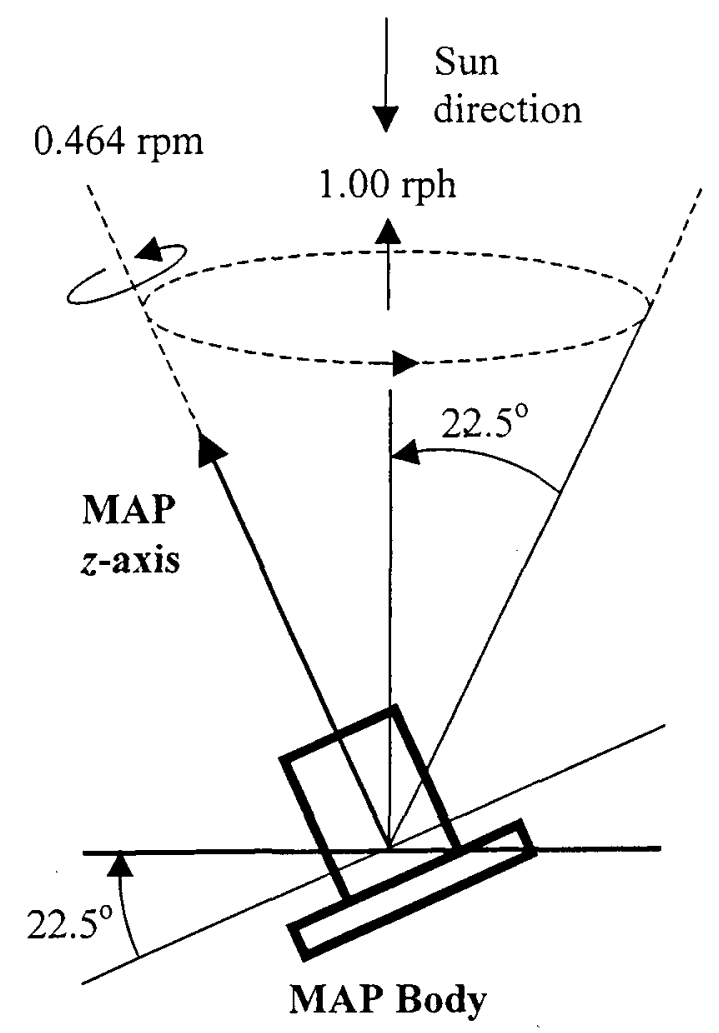

Fig. 1: The Geometry of the Angular Motion Components of the MAP Spacecraft.

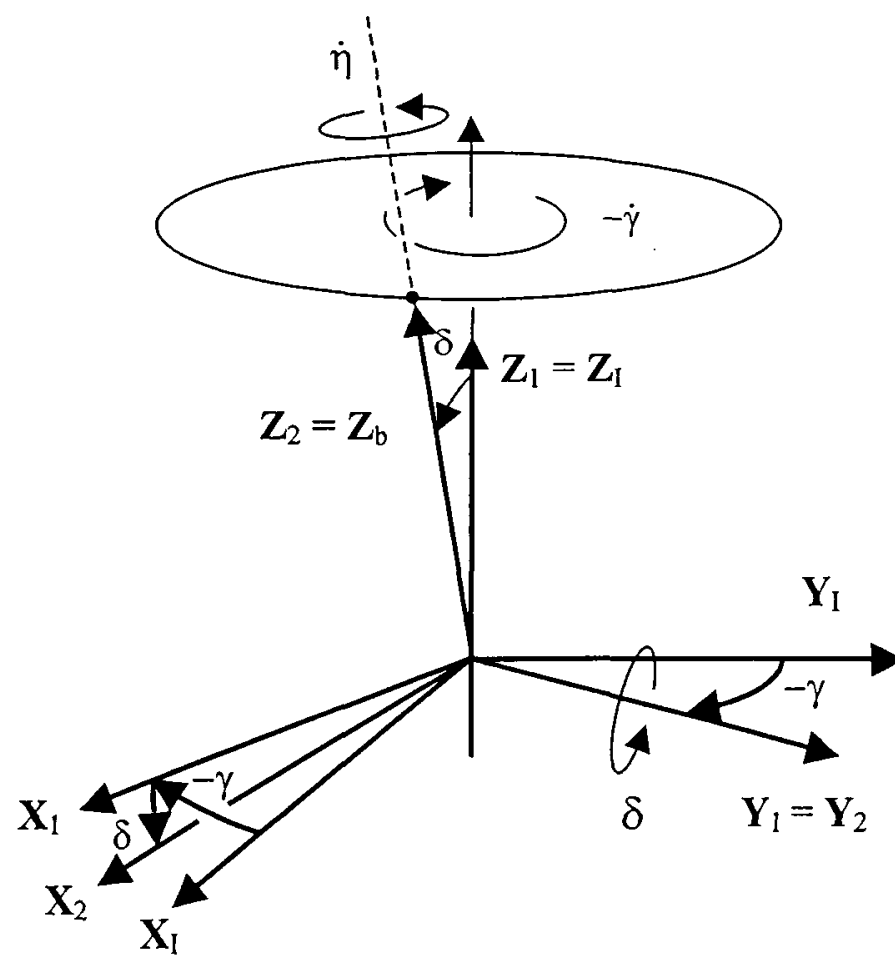

Fig. 2: Coordinate Systems Involved in the Description of the MAP Total Angular Velocity. angular rate profile. As a baseline rate profile we chose the MAP science collection rate profile at L2. At the L2 observation station the angular motion of MAP consists of two rotations as described in Fig. 1. One rotation, at 360 degrees an hour, is about the anti-Sun line, and the other, at $0.464 \mathrm{RPM}$, is about the satellite $z$-axis. The body $z$ axis is tilted by 22.5 degrees with respect to the anti-Sun line. The body angular rate and attitude definitions are presented in Fig. 2 in terms of three Euler angles. In order to describe the spacecraft motion and the associated measurements we define four coordinate systems. The first system is the Sun coordinate system (designated by sun). The center of this system is at the origin of the spacecraft coordinates, its $z$-axis points at the Sun direction and its $x$-axis, which is turning, is assumed to point at some arbitrary direction in space, which we define as zero $\gamma$. A rotation by the angle $-\gamma$ about the $z$-axis yields a first interim coordinate system, which we denote by 1 . A second rotation by the angle $\delta$ about the $y$-axis of this interim coordinate system yields the second interim coordinates, which we denote by 2 . Finally, a rotation by the angle $\eta$ about the $z$-axis of the latter system results in the body coordinates, which we denote by $b$. For sake of simplicity, only the body system z-axis is shown in Fig. 2. MAP rotates about two axes. One rotation, at the rate of $-\dot{\gamma}$, is about the Sun line, which we defined as the $\mathbf{Z}_{\text {sun }}$ axis, and the other, at the rate of $\dot{\eta}$, is about the body $z$-axis. Using Fig. (2) we obtain

$$
\begin{aligned}
& \omega_{x}=\dot{\gamma} \sin \delta \cdot \cos \eta \\
& \omega_{y}=-\dot{\gamma} \sin \delta \cdot \sin \eta \\
& \omega_{z}=-\dot{\gamma} \cos \delta+\dot{\eta}
\end{aligned}
$$

and the transformation matrix from the Sun to body coordinates is:

$$
\mathrm{D}_{\mathrm{b}}^{\text {sun }}=\left[\begin{array}{ccc}
c \gamma c \delta c \eta+s \gamma s \eta & -s \gamma c \delta c \eta+c \gamma s \eta & -\mathrm{s} \delta \mathrm{c} \eta \\
-\mathrm{c} \gamma c \delta s \eta+s \gamma c \eta & s \gamma c \delta s \eta+c \gamma c \eta & s \delta s \eta \\
c \gamma s \delta & -s \gamma \mathrm{s} \delta & \mathrm{c} \delta
\end{array}\right]
$$

where $s$ denotes the sine function and $c$ the cosine. The corresponding quaternion can be extracted best when using the newly derived algorithm. ${ }^{1}$ The angular rates and angles are as follows:

$$
\begin{aligned}
& \dot{\gamma}=1 \mathrm{rev} / \text { hour }=1 \cdot 2 \pi / 3600 \mathrm{rad} / \mathrm{sec} \\
& \gamma=\dot{\gamma} \mathrm{t}+0 \mathrm{rad}=\dot{\gamma} \mathrm{rad} \\
& \dot{\eta}=0.464 \mathrm{rpm}=0.464 \cdot 2 \pi / 60 \mathrm{rad} / \mathrm{sec}
\end{aligned}
$$

and

$$
\eta=\dot{\eta} t+0 \mathrm{rad}=\dot{\eta} t \mathrm{rad}
$$




$$
\delta=22.5^{\circ}=22.5 \cdot \pi / 180 \mathrm{rad}
$$

Upon examination of the MAP trajectory, the Sun direction and thus the Sun coordinates can be considered stationary in inertial space relative to the rotation of MAP with respect to the Sun coordinates. Consequently, we may consider the rate components of Eq. (1) as inertial rates resolved in body coordinates.

\section{Preliminary Analysis}

We wish to know the relationship between the sun sensor measurements and the angles $\gamma, \delta$ and $\eta$ that determine MAP's attitude. To do that we turn to Fig. 3 which depicts the orientation of the sun sensor boresight in body coordinates. The sun sensor reads the tangent of the angles $\alpha$ and $\beta$. We wish to express the readings of these tangents as functions of the angles $\gamma, \delta$ and $\eta$ defined in Fig. 2. To do that we define $\left(Z_{\text {sun }}\right)_{\mathrm{b}}$ and $\left(Z_{\text {sun }}\right)_{\text {sun }}$. The column matrix $\left(Z_{\text {sun }}\right)_{b}$ contains the components of the vector $\mathbf{Z}_{\text {sun }}$ when resolved in the body coordinates, and, similarly, the column matrix $\left(Z_{\text {sun }}\right)_{\text {sun }}$ contains the components of the vector $\mathbf{Z}_{\text {sun }}$ when resolved in the Sun coordinates. We denote the components of the former by $a_{x}, a_{y}$ and $a_{z}$. It is easy to see that the components of $\left(\mathbf{Z}_{\text {sun }}\right)_{\text {sun }}$ are 0,0 , and 1 . Thus

$$
\left(\boldsymbol{Z}_{\text {sun }}\right)_{\mathrm{b}}^{\mathrm{T}}=\left[\begin{array}{lll}
\mathrm{a}_{\mathrm{x}} & \mathrm{a}_{\mathrm{y}} & \mathrm{a}_{\mathrm{z}}
\end{array}\right]
$$

and

Obviously

$$
\left(\mathbf{Z}_{\text {sun }}\right)_{\text {sun }}^{\mathrm{T}}=\left[\begin{array}{lll}
0 & 0 & 1
\end{array}\right]
$$

$$
\left(\mathbf{Z}_{\text {sun }}\right)_{\mathrm{b}}=\mathrm{D}_{\mathrm{b}}^{\text {sun }}\left(\mathbf{Z}_{\text {sun }}\right)_{\text {sun }}
$$

From the last three equations and Eq. (2) we obtain

$$
\left[\begin{array}{l}
\mathrm{a}_{\mathrm{x}} \\
\mathrm{a}_{\mathrm{y}} \\
\mathrm{a}_{\mathrm{z}}
\end{array}\right]=\left[\begin{array}{cc} 
& -\mathrm{s} \delta \mathrm{c} \eta \\
\times & \mathrm{x} \delta \mathrm{s} \eta \\
& \mathrm{c} \delta
\end{array}\right]\left[\begin{array}{l}
0 \\
0 \\
1
\end{array}\right]
$$

hence

therefore

$$
\begin{aligned}
& a_{x}=-s \delta c \eta \\
& a_{y}=s \delta s \eta \\
& a_{z}=c \delta
\end{aligned}
$$

Note that $\tan \alpha$ and $\tan \beta$ are independent of $\gamma$. An inspection of Fig. 2 reveals that this is an expected result. Since $\tan \alpha$ and $\tan \beta$ are measured quantities, the last two equations contain only two unknowns, therefore we can solve for the angles $\delta$ and $\eta$. For this reason even if the measurements contain unbiased errors we can still find these two angles by proper filtration. In order to fully know the spacecraft attitude we need to know $\gamma$ too. If the gyros mounted on the Sun coordinates $z$-axis introduce no bias, then a proper integration of their measured outputs will yield $\gamma$ and thus we can obtain the spacecraft attitude from the measurements obtained from these gyros and the sun sensor. If the gyro measurements contain a bias then we ask ourselves whether the system is observable; that is, whether the spacecraft angular dynamics (Euler) equation may add information with which we can still observe $\gamma$ and thus completely determine the attitude. To answer this question we have to design an estimator and examine the observability of the system used by the estimator. This is done next.

\section{Filter I}

\section{THE FILTERS}

\section{Dynamics Model}

In this estimator (filter) we convert the sun sensors measurements to a vector measurement, which is then connected to the quaternion of the spacecraft. The dynamics model of Filter I was developed in. ${ }^{2}$ Using

$$
Q=\left[\begin{array}{ccc}
q_{4} & -q_{3} & q_{2} \\
q_{3} & q_{4} & -q_{1} \\
-q_{2} & q_{1} & q_{4} \\
-q_{1} & -q_{2} & -q_{3}
\end{array}\right]
$$

define the system dynamics as

$$
\left[\begin{array}{l}
\dot{\boldsymbol{\omega}} \\
\dot{\mathbf{q}}
\end{array}\right]=\left[\begin{array}{cc}
\mathrm{I}^{-1}[(\mathrm{I} \boldsymbol{\omega}+\mathbf{h}) \times] & 0 \\
\frac{1}{2} \mathrm{Q} & 0
\end{array}\right]\left[\begin{array}{l}
\mathbf{q} \\
\mathbf{q}
\end{array}\right]+\left[\begin{array}{c}
\mathrm{I}^{-1}(\mathbf{T}-\dot{\mathbf{h}}) \\
0
\end{array}\right]+\left[\begin{array}{l}
\mathbf{w}_{\omega} \\
\mathbf{w}_{\mathrm{q}}
\end{array}\right]
$$

where $[\mathbf{g} \times]$ is the cross product matrix of a general vector $g, \mathbf{w}_{\omega}$ accounts for the inaccuracies in the modeling of the spacecraft angular dynamics, and $\mathbf{w}_{\mathbf{q}}$ accounts for modeling errors of the quaternion dynamics.

\section{Measurement Equations}

The measurements are the tangent of the angles $\alpha$ and $\beta$, measured by the sun sensor and shown in Fig. 3, and the $z$ and either the $x$ or the $y$ component of the body rate measured by one of the spacecraft gyros. The measured quantities are contaminated by noise so they are not the 
reference values but rather, $\alpha_{\mathrm{m}}, \beta_{\mathrm{m}}, \omega_{z, \mathrm{~m}}$ and either $\omega_{\mathrm{x}, \mathrm{m}}$ or $\omega_{\mathrm{y}, \mathrm{m}}$, depending on which gyro failed.

\section{Sun Sensor Measurement}

Using the two measured angles we compute a unit vector in the assumed sun direction as follows ${ }^{3}$

$$
\mathbf{b}_{m}=\frac{1}{\sqrt{\tan \alpha_{m}^{2}+\tan \beta_{m}^{2}+1}}\left[\begin{array}{c}
\tan \beta_{m} \\
\tan \alpha_{m} \\
1
\end{array}\right]
$$

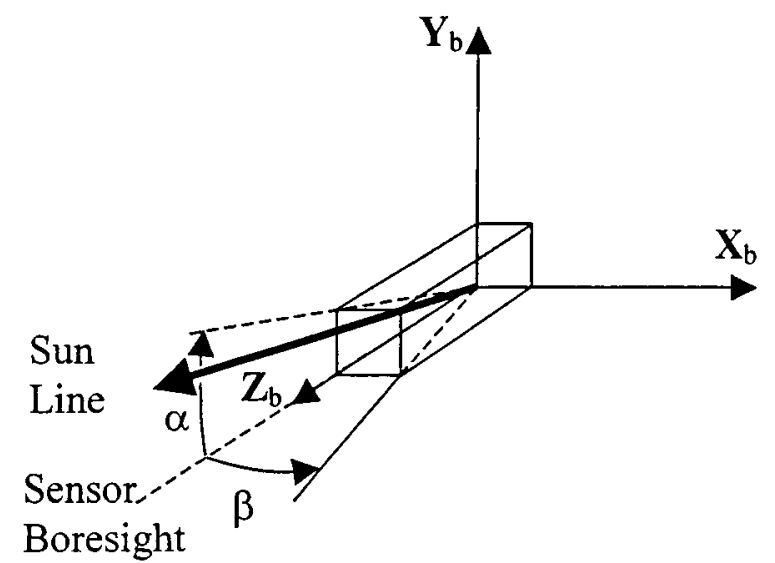

Fig. 3: Definition of the Sun Direction

In reality $\tan \beta_{\mathrm{m}}$ and $\tan \alpha_{\mathrm{m}}$ contain noise; however in the filter we approximate the $b_{m}$ measured vector as the true vector to the Sun and a simple additive zero mean white noise; that is,

$$
\mathbf{b}_{\mathrm{m}}=\mathbf{b}+\mathbf{v}_{\mathrm{b}}
$$

where $\mathbf{b}$ is the nominal unit vector in the true direction to the sun. The logic behind this simplification is that if in tests the filter yields satisfying results then the approximation is justified. Let $\mathbf{r}$ denote the measured Sun-vector expressed in the reference coordinate system (this vector is taken from the almanac). The relationship between the two vectors is expressed by

$$
\mathbf{b}=\mathrm{Dr}
$$

where $\mathrm{D}$ is the direction cosine matrix that transforms vectors from the reference to the body coordinates. From the last two equations we obtain

$$
\mathbf{b}_{\mathrm{m}}=\mathrm{Dr}+\mathbf{v}_{\mathrm{b}}
$$

It is well known that $\mathrm{D}$ is the following function of the quaternion elements ${ }^{4}$

$$
D=\left[\begin{array}{ccc}
q_{1}^{2}-q_{2}^{2}-q_{3}^{2}+q_{4}^{2} & 2\left(q_{1} q_{2}+q_{3} q_{4}\right) & 2\left(q_{1} q_{3}-q_{2} q_{4}\right) \\
2\left(q_{1} q_{2}-q_{3} q_{4}\right) & -q_{1}^{2}+q_{2}^{2}-q_{3}^{2}+q_{4}^{2} & 2\left(q_{2} q_{3}+q_{1} q_{4}\right) \\
2\left(q_{1} q_{3}+q_{2} q_{4}\right) & 2\left(q_{2} q_{3}-q_{1} q_{4}\right) & -q_{1}^{2}-q_{2}^{2}+q_{3}^{2}+q_{4}^{2}
\end{array}\right]
$$

when the last expression is substituted into Eq. (14) we obtain

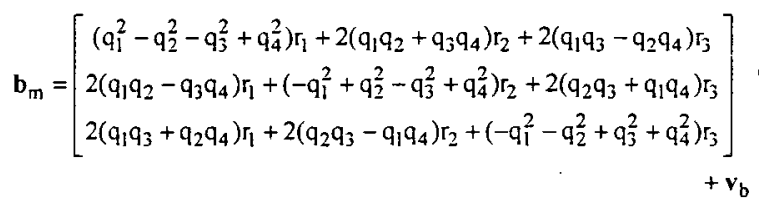

$$
\begin{aligned}
& \text { or } \\
& \qquad \begin{aligned}
b_{m}=\left[\begin{array}{c}
q_{1}^{2} r_{1} \\
q_{1} q_{2} r_{1}-q_{1} q_{2} r_{2}+q_{1} r_{2}+q_{3} r_{3} q_{4} r_{3}+q_{2}^{2} q_{2} r_{1} r_{1}+q_{1} q_{2} r_{2} r_{2}+q_{2} q_{3} q_{4} r_{3}-q_{3} q_{4} r_{1}^{2} r_{1} \\
q_{1} q_{3} r_{1}-q_{1} q_{4} r_{2}-q_{1}^{2} r_{3}+q_{2} q_{4} r_{1}+q_{2} q_{3} r_{2}-q_{2}^{2} r_{3}+q_{1} q_{3} r_{1} \\
+q_{3} q_{4} r_{2}+q_{1} q_{3} r_{3}+q_{4}^{2} r_{1}+q_{3} q_{4} r_{2}-q_{2} q_{4} r_{3} \\
-q_{3}^{2} r_{2}+q_{2} q_{3} r_{3}-q_{3} q_{4} r_{1}+q_{4}^{2} r_{2}+q_{1} q_{4} r_{3} \\
+q_{2} q_{3} r_{2}+q_{3}^{2} r_{3}+q_{2} q_{4} r_{1}-q_{1} q_{4} r_{2}+q_{4}^{2} r_{3}
\end{array}\right]+v_{b}
\end{aligned}
\end{aligned}
$$

which can be written as

$$
b_{m=}=\left[\begin{array}{rrr}
q_{1} r_{1}+q_{2} r_{2}+q_{3} r_{3} & -q_{2} r_{1}+q_{1} r_{2}-q_{4} r_{3} & -q_{3} r_{1}+q_{4} r_{2}+q_{1} r_{3} \\
q_{2} r_{1}-q_{1} r_{2}+q_{4} r_{3} & q_{1} r_{1}+q_{2} r_{2}+q_{3} r_{3} & -q_{4} r_{1}-q_{3} r_{2}+q_{2} r_{3} \\
q_{3} r_{1}-q_{4} r_{2}-q_{1} r_{3} & q_{4} r_{1}+q_{3} r_{2}-q_{2} r_{3} & q_{1} r_{1}+q_{2} r_{2}+q_{3} r_{3} \\
& q_{4} r_{1}+q_{3} r_{2}-q_{2} r_{3} \\
-q_{3} r_{1}+q_{4} r_{2}+q_{1} r_{3} \\
q_{2} r_{1}-q_{1} r_{2}+q_{4} r_{3}
\end{array}\right]\left[\begin{array}{l}
q_{1} \\
q_{2} \\
q_{3} \\
q_{4}
\end{array}\right]+v_{b}
$$

Let

$$
\begin{aligned}
& H_{b}(r, q)=\left[\begin{array}{cc}
q_{1} r_{1}+q_{2} r_{2}+q_{3} r_{3} & -q_{2} r_{1}+q_{1} r_{2}-q_{4} r_{3} \\
q_{2} r_{1}-q_{1} r_{2}+q_{4} r_{3} & q_{1} r_{1}+q_{2} r_{2}+q_{3} r_{3} \\
q_{3} r_{1}-q_{4} r_{2}-q_{1} r_{3} & q_{4} r_{1}+q_{3} r_{2}-q_{2} r_{3}
\end{array}\right. \\
& \left.\begin{array}{cc}
-q_{3} r_{1}+q_{4} r_{2}+q_{1} r_{3} & q_{4} r_{1}+q_{3} r_{2}-q_{2} r_{3} \\
-q_{4} r_{1}-q_{3} r_{2}+q_{2} r_{3} & -q_{3} r_{1}+q_{4} r_{2}+q_{1} r_{3} \\
q_{1} r_{1}+q_{2} r_{2}+q_{3} r_{3} & q_{2} r_{1}-q_{1} r_{2}+q_{4} r_{3}
\end{array}\right]
\end{aligned}
$$

then Eq. (18) can be written as

$$
\mathbf{b}_{\mathrm{m}}=\mathrm{H}_{\mathrm{b}}(\mathbf{r}, \mathbf{q}) \mathbf{q}+\mathbf{v}_{\mathrm{b}}
$$

or

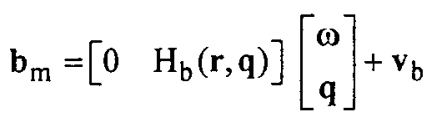

Eq. (21) is the measurement equation associated with the sun sensor measurement. 
Gyro Measurement

Let us assume that the gyro which measures rates along the $x$ and $z$ axes continues to operate. The $x$ and $z$ axes rate measurements can be written as

$$
\begin{aligned}
& \omega_{x, m}=\omega_{x}+v_{\omega x} \\
& \omega_{z, m}=\omega_{z}+v_{\omega z}
\end{aligned}
$$

where $\omega_{\mathrm{x}}$ and $\omega_{\mathrm{z}}$ are the true rates, and $\mathrm{v}_{\omega \mathrm{x}}$ and $\mathrm{v}_{\omega \mathrm{z}}$ are scalar zero-mean white-noise measurements. Define

$$
H_{\omega}=\left[\begin{array}{lll}
1 & 0 & 0 \\
0 & 0 & 1
\end{array}\right]
$$

then from the last two equations we obtain

$$
\left[\begin{array}{l}
\omega_{x, m} \\
\omega_{z, m}
\end{array}\right]=\left[\begin{array}{ll}
H_{\omega} & 0 \\
2 x^{4}
\end{array}\right]\left[\begin{array}{l}
\omega \\
q
\end{array}\right]+\left[\begin{array}{l}
v_{\omega x} \\
v_{\omega z}
\end{array}\right]
$$

We denote the measurement vector by $\omega_{\mathrm{m}}$ where, clearly, $\boldsymbol{\omega}_{\mathrm{m}}^{\mathrm{T}}=\left[\begin{array}{ll}\omega_{\mathrm{x}, \mathrm{m}} & \omega_{z, \mathrm{~m}}\end{array}\right]$. Similarly we denote the noise vector as by $\mathbf{v}_{\omega}$ where $\mathbf{v}_{\omega}^{T}=\left[\begin{array}{ll}v_{\omega x} & v_{\omega z}\end{array}\right]$. Obviously, if the other gyro is the one that is still operating, then the $x$ component in Eqs. $(22-24)$ has to be replaced by the $y$ component.

\section{The Combined measurement}

Combining Eqs. (21) and (24) we obtain

$$
\left[\begin{array}{l}
\mathbf{b}_{\mathrm{m}} \\
\omega_{\mathrm{m}}
\end{array}\right]=\left[\begin{array}{cc}
0_{3 \times 3} & \mathrm{H}_{\mathrm{b}}(\mathbf{r}, \mathbf{q}) \\
\mathrm{H}_{\omega} & 0_{2 \times 4}
\end{array}\right]\left[\begin{array}{l}
\omega \\
\mathbf{q}
\end{array}\right]+\left[\begin{array}{l}
\mathbf{v}_{\mathrm{b}} \\
\mathbf{v}_{\omega}
\end{array}\right]
$$

This is the combined measurement model for simultaneous measurement of the operating two-axes gyro and the sun sensor.

\section{Filter II}

\section{Dynamics Model}

In this filter we make use of the kinematics rule governing the rate of change of the Sun vector. It is known that

$$
D \dot{r}=\dot{b}+\omega \times b
$$

hence

$$
\dot{\mathbf{b}}=[\mathbf{b} \times] \omega+D \dot{\mathbf{r}}
$$

As mentioned earlier, the vector $\mathbf{r}$ is known from the almanac, therefore $\dot{\mathbf{r}}$ is known too. Consequently we can treat $D \dot{\mathbf{r}}$ as a deterministic forcing function. In our case $r$ is the vector from the spacecraft to the Sun. For the MAP trajectory this vector changes very slowly. Therefore we can neglect the term in $\dot{\mathbf{r}}$ and use

$$
\dot{b}=[\mathbf{b} \times] \omega
$$

Augmenting Eq. (26.c) with the dynamics of Filter I yields

$$
\begin{aligned}
{\left[\begin{array}{c}
\dot{\boldsymbol{\omega}} \\
\dot{\mathbf{q}} \\
\dot{\mathbf{b}}
\end{array}\right]=\left[\begin{array}{ccc}
\mathrm{I}^{-1}[(\mathrm{I} \boldsymbol{\omega}+\mathbf{h}) \times] & 0 & 0 \\
\frac{1}{2} \mathrm{Q} & 0 & 0 \\
{[\mathrm{~b} \times]} & 0 & 0
\end{array}\right]\left[\begin{array}{l}
\boldsymbol{\omega} \\
\mathbf{q} \\
\mathbf{b}
\end{array}\right] } \\
+\left[\begin{array}{c}
\mathrm{I}^{-1}(\mathbf{T}-\dot{\mathbf{h}}) \\
0 \\
\mathrm{Dr}
\end{array}\right]+\left[\begin{array}{l}
\mathbf{w}_{\mathrm{\omega}} \\
\mathbf{w}_{\mathrm{q}} \\
\mathbf{w}_{\mathrm{b}}
\end{array}\right]
\end{aligned}
$$

The state vector in Eq. (27) includes the Sun vector $\mathbf{b}$ even though $\mathbf{b}$ is a measurement. Indeed, in order to formally compute the rate and attitude one can numerically differentiate the measured $b_{x}$ and $b_{y}$, and use them in the following equations (which are the components of Eq. (26.c))

$$
\begin{aligned}
& \dot{b}_{x}=\omega_{y} b_{x}-\omega_{x} b_{y} \\
& \dot{b}_{y}=\omega_{z} b_{x}-\omega_{x} b_{z}
\end{aligned}
$$

together with the gyro-measured $\omega_{z}$, to solve for the two unknowns $\omega_{\mathrm{x}}$ and $\omega_{\mathrm{y}}$. Then use these $\omega_{\mathrm{x}}$ and $\omega_{\mathrm{y}}$ and the measured $\omega_{z}$ as 'measurements' in an estimator whose dynamics model consists of the first two matrix equations in Eq. (27). However, this method required numerical differentiation, which introduces noise. To avoid differentiation we apply the estimation approach. ${ }^{5}$ The latter can be accomplished by adding Eq. (26.c) to Eq. (10), the differential equations of the variables that we want to estimate, thus forming Eq. (27). At this point a Kalman filter can be utilized to estimate those variables.

\section{Measurement Equations}

The measurement equations of Filter II are quite simple. The vector measurement of Eq. (21) is replaced by the simple equation

$$
\mathbf{b}_{\mathrm{m}}=\left[\begin{array}{lll}
0 & 0 & I_{3}
\end{array}\right]\left[\begin{array}{l}
\omega \\
\mathbf{q} \\
\mathbf{b}
\end{array}\right]+\mathbf{v}_{\mathrm{b}}
$$

Eq.(24) stays the same; therefore, the augmented measurement equation for Filter II becomes

$$
\left[\begin{array}{l}
\mathbf{b}_{m} \\
\omega_{m}
\end{array}\right]=\left[\begin{array}{ccc}
0 & 0 & I_{3} \\
H_{\omega} & 0 & 0
\end{array}\right]\left[\begin{array}{l}
\omega \\
q \\
b
\end{array}\right]+\left[\begin{array}{c}
\mathbf{v}_{b} \\
\mathbf{v}_{\omega}
\end{array}\right]
$$

\section{OBSERVABILITY TESTS}

Before carrying on an observability test it is necessary to specify the kind of filter that was used in this analysis. The filter that was used here is the PSELIKA (PseudoLinear Kalman) Filter. ${ }^{6}$ The idea on which this filter is based is as follows. Suppose that the non-linear dynamics 
and measurement equations of a non-linear system can be decomposed in the following way

$$
\begin{aligned}
& \dot{\mathbf{x}}=\mathrm{F}(\mathbf{x}) \mathbf{x}+\mathrm{Bu}+\mathbf{w} \\
& \mathbf{y}=\mathrm{H}(\mathbf{x}) \mathbf{x}+\mathbf{v}
\end{aligned}
$$

If $F(\hat{\mathbf{x}})$ is close enough to $F(\mathbf{x})$ and $H(\hat{\mathbf{x}})$ is close enough to $H(\mathbf{x})$ (note that we do not assume that $\hat{\mathbf{x}}$ is necessarily close to $\mathbf{X}$ ) then instead of the unknown matrices $F(\mathbf{x})$ and $H(\mathbf{x})$ we can use respectively $F(\hat{\mathbf{x}})$ and $H(\hat{\mathbf{x}})$ which are known along the estimation process and apply the linear Kalman filter algorithm to the measurements using the models

$$
\begin{aligned}
& \dot{\mathbf{x}}=F(\hat{\mathbf{x}}) \mathbf{x}+\mathrm{Bu}+\mathbf{w} \\
& \mathbf{y}=H(\hat{\mathbf{x}}) \mathbf{x}+\mathbf{v}
\end{aligned}
$$

The observability analysis requires $\mathrm{F}(\mathbf{x})$ and $\mathrm{H}(\mathbf{x})$. The $\mathbf{x}$ for $F(x)$ and $H(x)$ was the simulation truth model state. The logic for this choice of $\mathbf{x}$ is as follows. If when using $F(\mathbf{x})$ and $H(x)$ in the analysis the latter indicates that the system is not observable under these favorable conditions then there is no sense in trying to estimate the state vector $\mathbf{x}$ of the system. If, on the other hand, the analysis indicates that the system is observable, then although observability and therefore estimability are not assured, it makes sense to design an estimator and attempt to estimate $\mathbf{x}$. The observability of the system models used by the two filters was examined using the following analysis. Consider $\Phi_{k}$, the transition matrix, which corresponds to F, the dynamics matrix, of either Eq. (10) or Eq. (27). This matrix transforms the system state vector at time $t_{k}, x_{k}$, to $\mathbf{x}_{k+1}$, the state at time $t_{k+1}$. If at a certain time-point, $t_{m}$, the initial state, denoted by $\mathbf{x}_{0}$, can be computed, then, for our purposes, the system is observable. Adopting the common approach to proving complete observability of a discrete linear system, we express the first $m$ measurements as follows

$$
\mathbf{y}_{j}=H_{j}\left(\mathbf{x}_{j}\right)\left[\prod_{i=0}^{j-1} \Phi_{i}\left(x_{i}\right)\right] x_{0} \quad j=0,1, \ldots, m
$$

where $\Phi_{0}\left(\mathbf{x}_{0}\right)=\mathrm{I}$, then the last equation can be written as

$$
\mathbf{Y}=\mathbf{W} \mathbf{x}_{0}
$$

where

$$
\mathbf{Y}=\left[\begin{array}{lllll}
\mathbf{y}_{1} & \mathbf{y}_{2} & . & \mathbf{y}_{\mathrm{m}}
\end{array}\right]^{\mathrm{T}}
$$

and

$$
\mathbf{W}=\left[\begin{array}{c}
\mathrm{H}_{0}\left(\mathbf{x}_{0}\right) \\
\mathrm{H}_{1}\left(\mathbf{x}_{1}\right) \Phi_{0}\left(\mathbf{x}_{0}\right) \\
\mathrm{H}_{2}\left(\mathbf{x}_{2}\right) \Phi_{1}\left(\mathbf{x}_{1}\right) \Phi_{0}\left(\mathbf{x}_{0}\right) \\
\cdot \\
\cdot \\
H_{m}\left(\mathbf{x}_{\mathrm{m}}\right) \Phi_{\mathrm{m}-1}\left(\mathbf{x}_{\mathrm{m}-1}\right) \cdots \Phi_{0}\left(\mathbf{x}_{0}\right)
\end{array}\right]
$$

Note that in Filter II, H is not a function of the state (see equations 23 and 30 ). That is, the measurement equation is linear from the start. If there are $n$ independent rows in the right hand side matrix (the observability matrix) in Eq. (32) where $n$ is also the number of states, then $\mathbf{x}_{0}$ can be solved for and the state is observable. The observability

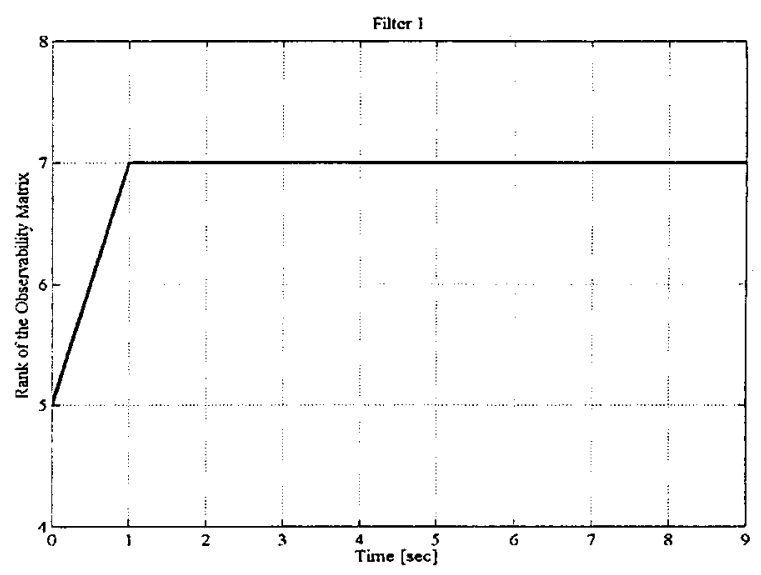

Fig. 4: Rank Development of the Observability Matrix of Filter I.

test of Filter I reveals that the rank of the observability matrix for this filter becomes 7 (see Fig. 4), and since the filter state is 7 too, the system is observable. It means that there is a good chance that Filter I can estimate both the

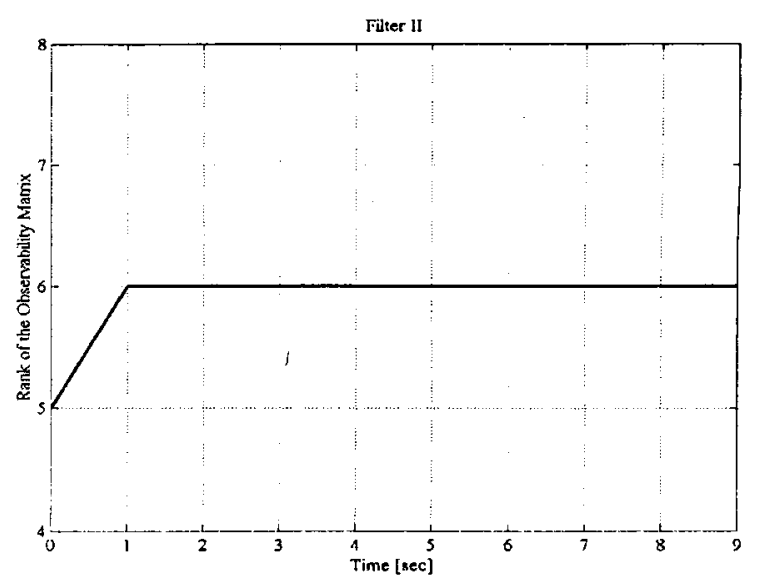

Fig. 5: Rank Development of the Observability Matrix of Filter II. 
missing angular rate component as well as the attitude of the spacecraft when one gyro fails. We note though that the measurement equation of Filter I is only pseudo-linear and not truly linear. This fact may cause a problem even though the observability test indicates that the state is completely observable. The observability matrix of Filter II becomes 6 and stay at 6 (see Fig. 5) whereas the size of the state vector is 9. This disparity indicates that the system is not completely observable. It means that we cannot fully estimate the missing rate component and the attitude. However, maybe their estimated values are close enough to the truth. Therefore both filters had to be tested. Simulation results of the two filters are presented in the next section.

\section{SIMULATION TEST RESULTS}

Simulations were run for different initial conditions and noise levels. Plots that resulted from a typical run of Filter I are presented in Figs. $6-11$. For this run the initial $\gamma$ angle
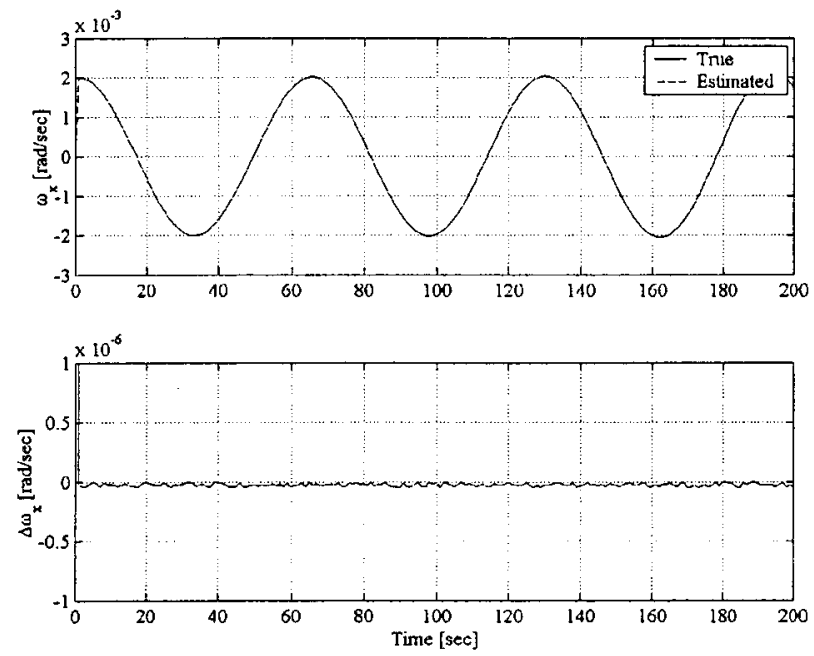

Fig. 6: The $x$-component of the angular velocity, its estimate, and its estimation error for Filter I.
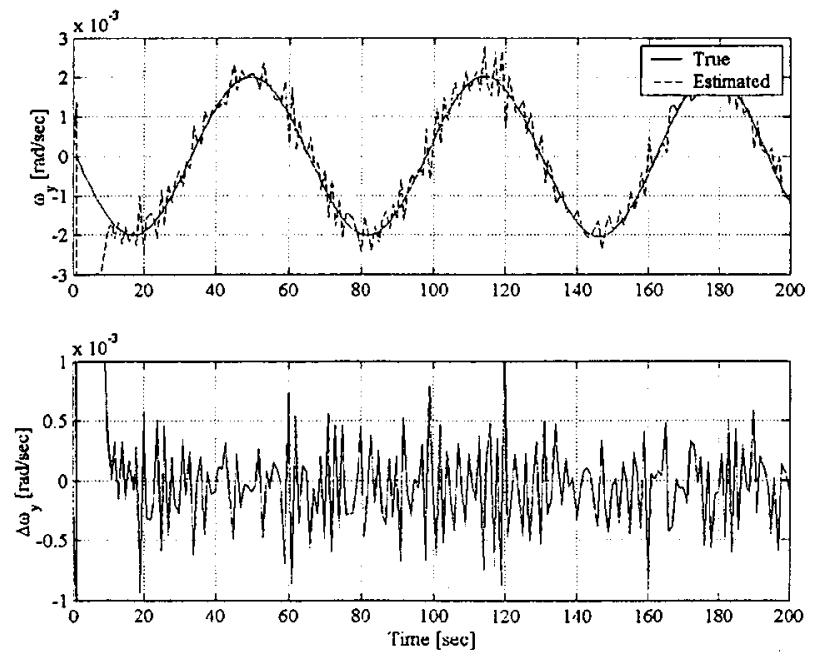

Fig. 7: The $y$-component of the angular velocity, its estimate, and its estimation error for Filter I.
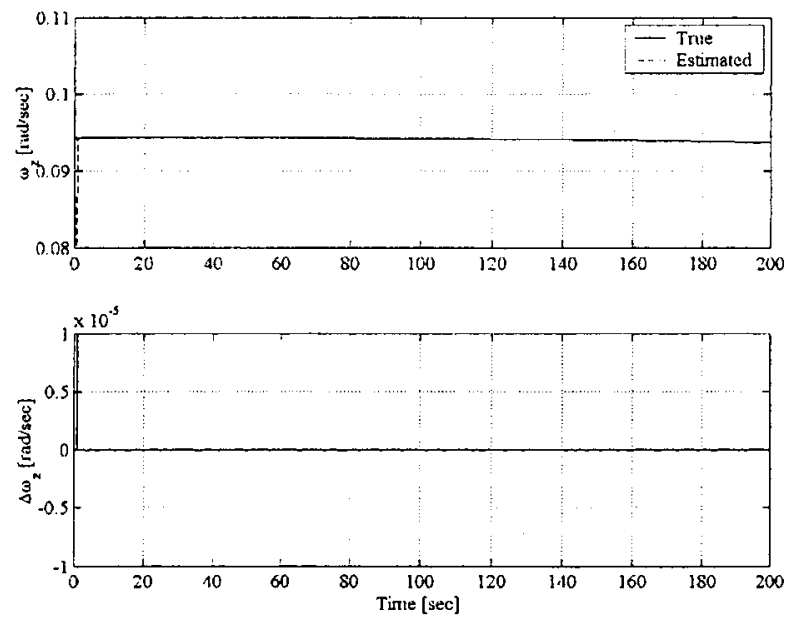

Fig. 8: The $z$-component of the angular velocity, its estimate, and its estimation error for Filter I.

was 60 degrees, $\delta$ was 35 degrees and $\eta$ was 0 . The initial estimates were zero for all three angles. The true Euler-angle rates were $2 \cdot(2 \pi) / 3600 \mathrm{rad} / \mathrm{sec}$ for $\dot{\gamma}$, zero for $\dot{\delta}$ and $0.928 \cdot(2 \pi) / 60 \mathrm{rad} / \mathrm{sec}$ for $\dot{\eta}$. According to Eq. (1), using these initial rates and angles, the true initial angular rate components were as follows: $\omega_{x}=0.002 \mathrm{rad} / \mathrm{sec} \omega_{y}=0 \mathrm{rad} / \mathrm{sec}$ and $\omega_{z}=0.094$ $\mathrm{rad} / \mathrm{sec}$. The initial estimated values of the angular velocity were zero for all three components. The onesigma gyro measurement noise was $0.01 \mathrm{deg} / \mathrm{sec}$ and that of the sun sensor was 0.25 degrees. In each figure we see the true state superimposed on the estimate of the state. Underneath this plot there is a plot of the estimation error. In this run $\gamma$ is estimated quite well, but this is not always the case. The observability of this angle is quite poor.
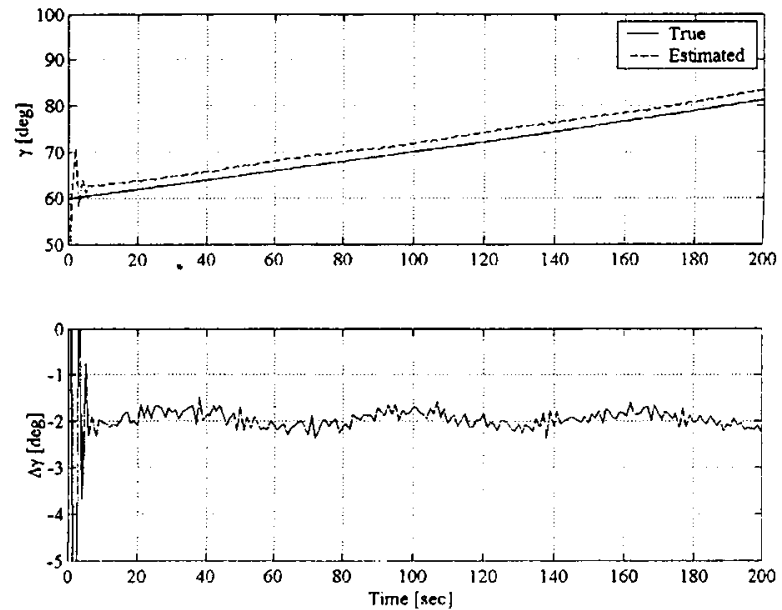

Fig. 9: The $\gamma$ angle, its estimate, and the estimation error for Filter 1 . 

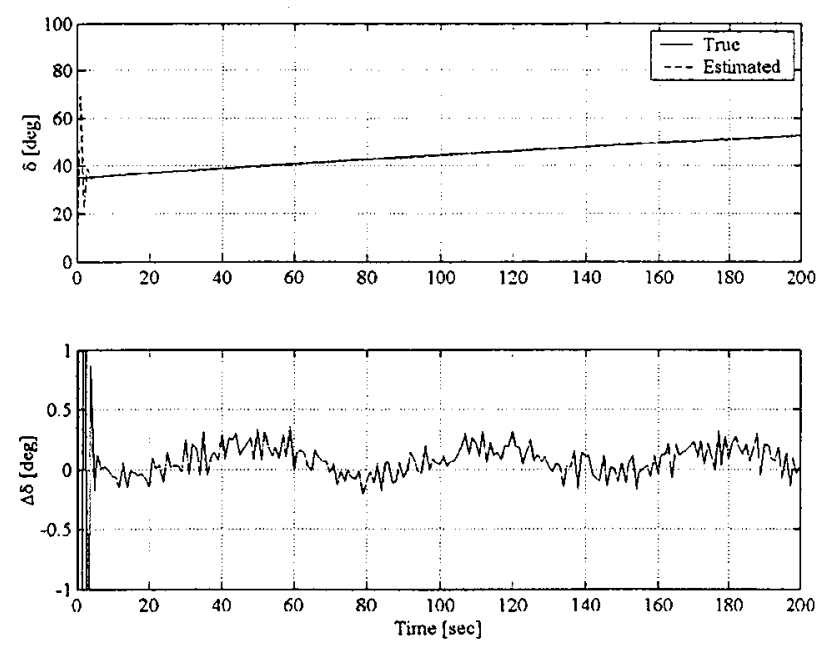

Fig. 10: The $\delta$ angle, its estimate, and the estimation error for Filter I.
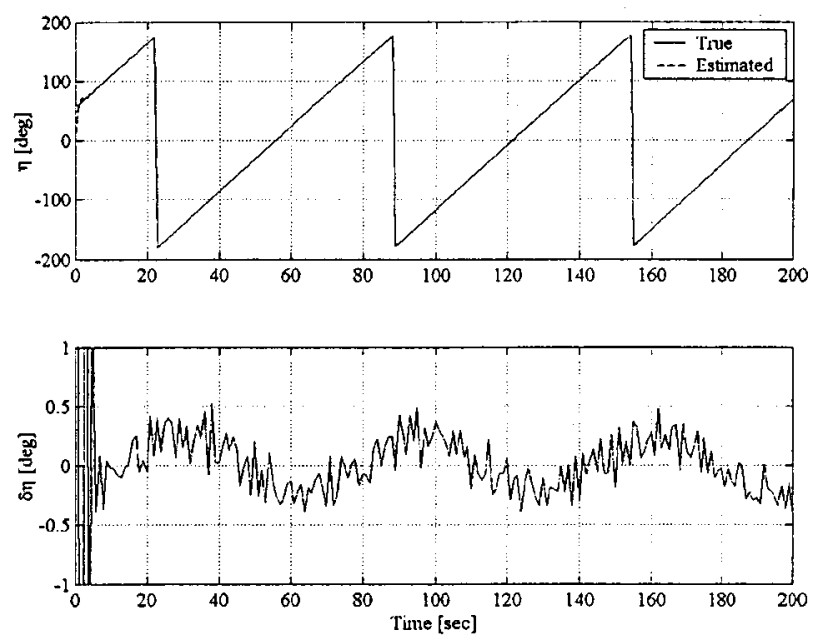

Fig. 11: The $\eta$ angle, its estimate, and the estimation error for Filter I.

Filter II was also run but, as expected from the observability test, the three attitude angles were not observable. The estimates of the angular rate components resemble those obtained using Filter I but the estimates of the three angles were quite poor as seen in Figs. 12-14.

\section{CONCLUSIONS}

In this paper we analyzed two algorithms for estimating the attitude and rate of the MAP spacecraft after one of its two two-degree of freedom and one of its vector-measuring devices fail. Both algorithms implement Kalman filters for estimating its attitude and rate. A special observability analysis that was carried out revealed that one of the filters, Filter I, was completely observable whereas the other filter, Filter II, was not completely observable. It was confirmed through simulations, which used the MAP spacecraft attitude and rate profile at the L2 Lagrange point, that Filter II was indeed unable to estimate the attitude. However, it
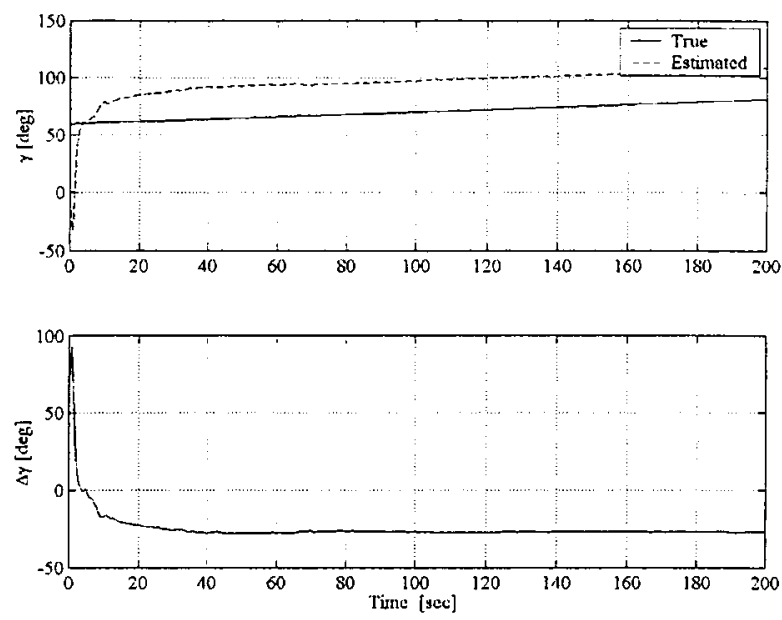

Fig. 12: The $\gamma$ angle, its estimate, and the estimation error for Filter II.
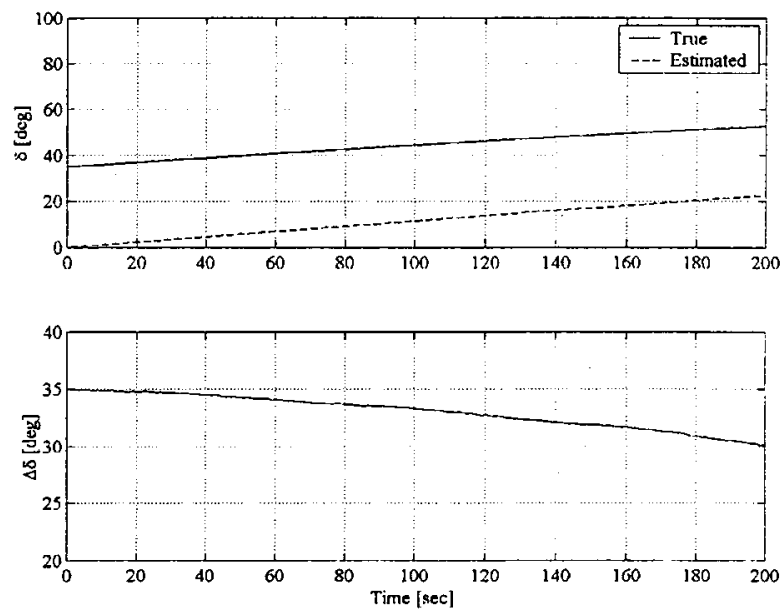

Fig. 13: The $\delta$ angle, its estimate, and the estimation error for Filter II.
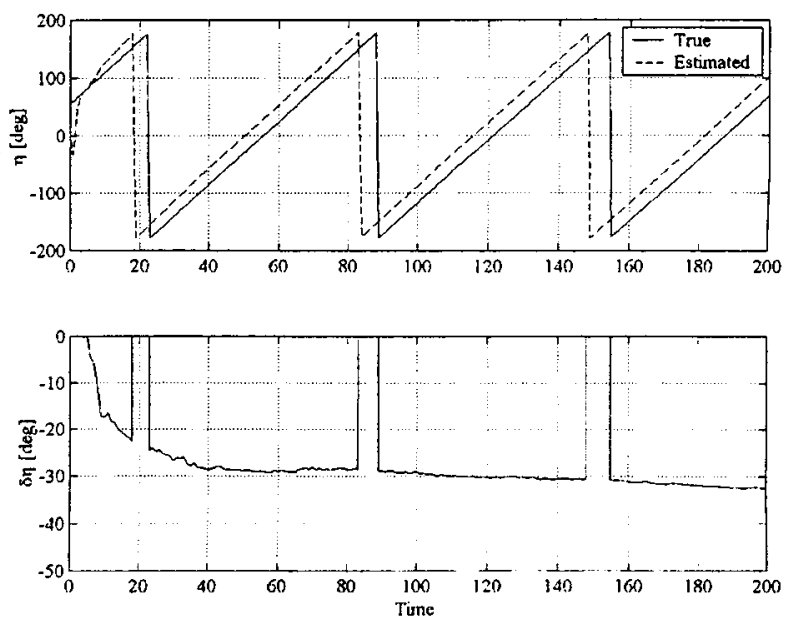

Fig. 14: The $\eta$ angle, its estimate, and the estimation error for Filter II. 
was also found that, contrary to the results of the observability analysis, when using Filter $I$ the estimation quality of one of the attitude angles depended on the initial errors. A possible explanation to this contradiction is based on the fact that the observability matrix that was used to examine the observability of the filter was state dependent. Therefore only when we ran the test using the correct state; namely, the nominal angular rate vector and Euler angles, was the system found to be observable. In reality, however, the existing state vector was the estimated state vector. Therefore as long as the initial estimate was close enough to the true state, the simulation conditions matched those of the analysis and the observability test results predicted well the performance of the filter. On the other hand, when the initial estimate of the state vector was far from the true vector the analysis was not valid anymore. The main conclusion of this analysis is that when only one vector-measuring sensor is active, MAP dynamics equations cannot assist in turning the system into a completely observable system. Therefore complete attitude estimation is impossible under the conditions described in the paper.

\section{Acknowledgement}

The authors wish to thank Mr. Mark Koifman of the Philadelphia Flight Control Laboratory of the Technion Aerospace Faculty for performing the simulation runs.

\section{REFERENCES}

1 Bar-Itzhack, I.Y., "New Method for Extracting the Quaternion from a Rotation Matrix", $A I A A J$. of Guidance, Control, and Dynamics, Vol. 23, No. 6, Nov.-Dec. 2000, pp. 1085-1087.

2 Azor, A., I.Y. Bar-Itzhack, J. Deutschmann and R.R. Harman, "Angular-Rate Estimation Using Delayed Quaternion Measurements", AIAA J. of Guidance, Control, and Dynamics, AIAA J. of Guidance, Control, and Dynamics, Vol. 24, No. 3, May-June 2001, pp. 436443.

${ }^{3}$ Wertz, J. R., (Ed.), Spacecraft Attitude Determination and Control, D. Reidel, Dordrecht, The Netherlands, 1984. p. 226.

4 Ibid, p. 414.

5 Bar-Itzhack, I.Y., "Classification of Algorithms for Angular Velocity Estimation", AIAA J. of Guidance, Control, and Dynamic, Vol. 24, No. 2, March-April 2001, pp. 214-218.

${ }^{6}$ Harman, R.R. and Bar-Itzhack, I.Y., "Pseudolinear and State-Dependent Riccati Equation Filters for Angular Rate Estimation", AlAA J. of Guidance, Control, and Dynamics, Vol. 22, No. 5, Sept.-Oct. 1999, pp. 723-725. (Engineering Note). 